\title{
Neural Response to Trauma-Related and Trauma- Unrelated Negative Stimuli in Remitted and Persistent Pediatric Posttraumatic Stress Disorder
}

\section{Peng Wang}

Peking University Institute of Mental Health: Peking University Sixth Hospital

\section{Zulai Peng}

Peking University Institute of Mental Health: Peking University Sixth Hospital

\section{Lu Liu}

Peking University Institute of Mental Health: Peking University Sixth Hospital

\section{Li An}

Peking University Institute of Mental Health: Peking University Sixth Hospital

\section{Yuxin Liu}

Peking University Institute of Mental Health: Peking University Sixth Hospital

\section{Qingjiu Cao}

Peking University Institute of Mental Health: Peking University Sixth Hospital

\section{Li Sun}

Peking University Institute of Mental Health: Peking University Sixth Hospital

Ning Ji

Peking University Institute of Mental Health: Peking University Sixth Hospital

\section{Yun Chen}

Peking University Institute of Mental Health: Peking University Sixth Hospital

\section{Binrang Yang}

Peking University Institute of Mental Health: Peking University Sixth Hospital

\section{Yufeng Wang ( $\nabla$ wangyf@bjmu.edu.cn )}

Peking University Institute of Mental Health: Peking University Sixth Hospital https://orcid.org/00000003-0831-3186

\section{Research}

Keywords: PTSD, fMRI, posttraumatic

Posted Date: October 16th, 2020

DOI: https://doi.org/10.21203/rs.3.rs-92060/v1 
License: (c) (i) This work is licensed under a Creative Commons Attribution 4.0 International License. Read Full License 


\section{Abstract}

Background: Most youths who suffer from posttraumatic stress disorder (PTSD) lose their diagnosis in the first 1-2 years. However, there are few studies on this brain mechanism, and the heterogeneity of the findings is partially due to the different stimuli applied and the mixed trauma history. Therefore, the use of trauma-related/unrelated stimuli to study the remittance mechanism of earthquake-induced PTSD could advance our knowledge of PTSD and inspire future treatment.

Methods: Thirteen youths with PTSD, 18 remitted participants and 18 control participants underwent functional magnetic resonance imaging (fMRI) while viewing trauma-related pictures, trauma-unrelated negative pictures and scrambled pictures.

Results: Under trauma-unrelated condition, the neural activity of the left hippocampus in the remitted group was in the middle between the two other groups. Under trauma-related condition, the PTSD and the remitted group exhibited higher neural activity in the right middle occipital gyrus than controls. The remitted group showed higher neural activity in the right parahippocampal gyrus and right lingual gyrus under trauma-related condition than trauma-unrelated condition, while no significant difference was found in PTSD group.

Conclusion: PTSD symptom-related group difference is mainly reflected in the left hippocampus under the trauma-unrelated condition, while the hyperactivity in the right middle occipital gyrus under trauma related condition could be an endophenotype for PTSD.

\section{Background}

Natural disasters always leave large numbers of young survivors suffering from various mental disorders, with posttraumatic stress disorder (PTSD) being the most common.(Zhang et al., 2015) The prevalence of PTSD in youths after disasters ranges from 2.5-60.0\%.(Tang, Deng, Glik, Dong, \& Zhang, 2017) Reexperiencing/intrusion, avoidance/emotional numbing, cognition/mood negative alterations and hypervigilance are the defining symptoms of PTSD.(Bovin, Marx, \& Schnurr, 2015)

Without specific treatment, most adolescents with PTSD spontaneously recover after an average of 14.8 months, but approximately one-third of patients show sustained psychopathology.(McLaughlin et al., 2013) A recent study of the Chinese population also showed that the prevalence of PTSD in youths was $43.9 \%$ twelve months after an earthquake, and it dropped to $15.7 \%$ at 30 months without treatment.(W. Tang et al., 2017) The data from an adult study were similar to those for youths, and these data showed that PTSD was sustained for several years in approximately one-third of PTSD patients.(Rosellini et al., 2018) The brain mechanism of PTSD remission is important to reveal the pathological mechanism of PTSD and the development/improvement of therapies. Research on the PTSD remittance mechanism has primarily focused on adults, while studies on youths are rare. 
Malejko et al. recently reviewed 19 longitudinal studies of PTSD (17 adult and 2 youth) and found that the remittance of PTSD was related to decreased activities of the insula and amygdala and increased activities of the prefrontal/anterior cingulate cortex (PFC/ACC) and hippocampus.(Malejko, Abler, Plener, \& Straub, 2017) Based on the ratio of the above studies, there has been relatively little PTSD-remittance research on youths. In 2016, Cisler et al. applied trauma-unrelated emotional stimuli to female adolescents with PTSD ('direct physical assault/sexual assault/witnessed violence') and found several findings. 1. Adolescents with less symptom reduction were characterized by less threat-safety discrimination before treatment (i.e., greater amygdala activation to both threat and neutral images), whereas adolescents with greater symptom reduction were characterized by amygdala activation only to threat images(Cisler et al., 2015) (included in the aforementioned review(Malejko et al., 2017)). 2. The recovery of adolescent PTSD was positively related to the functional connectivity between the right amygdala and insula and the left amygdala and posterior cingulate gyrus(Cisler, Sigel, Steele, et al., 2016) (included in the aforementioned review(Malejko et al., 2017)). 3. The remittance of PTSD was related to the high modularity and assortativity of the whole-brain network.(Cisler, Sigel, Kramer, et al., 2016) Another recent study was performed on youths with PTSD caused by interpersonal traumas ('physical abuse/sexual abuse/witnessing violence/other maltreatment-related traumas') and a control group without trauma experience. The study used a facial expression task (trauma unrelated) and found that the remittance was related to reduced activation of the posterior cingulate, mid-cingulate and hippocampus.(Garrett et al., 2019)

However, the heterogeneity found in previous studies is one of the important concerns in this field (e.g., in the review mentioned above, differences in the hippocampus appeared in only three of the 19 reviewed studies, two of these three studies showed increased activation, and one study showed decreased activation).(Malejko et al., 2017) The frequently discussed possible factors that could affect the heterogeneity of findings include trauma history, stimulation paradigm (trauma related/unrelated), and the control group (whether trauma exposed).(Malejko et al., 2017; Negreira \& Abdallah, 2019) These factors should be seriously considered when designing PTSD experiments and interpreting the results. In the study of youths, although there was some overlap with findings in adult studies, heterogeneity between the results was still observed.

Overall, the neurofunctional recovery mechanism of PTSD in youths is not clear. Among the factors that could contribute to heterogeneity in the above studies, mixed trauma history among the participants may be one of the most important because the abnormal brain activity caused by various traumatic events was different.(Boccia et al., 2016) The use of participants with the same trauma history may make the research more focused. Moreover, it also makes it possible to apply a unified trauma-related stimulus across all participants, including trauma-exposed controls. As mentioned above, the experimental paradigms (trauma related/unrelated) and control group (whether trauma exposed) are also important factors for heterogeneity. Considering the high heterogeneity across previous findings, these factors must have complex effects on the pathological mechanism of PTSD and are worthy of study. Most of the adult studies and all of the youth studies on PTSD-remittance mechanisms used trauma-unrelated stimuli. (Garrett et al., 2019; Malejko et al., 2017) However, trauma-related/unrelated stimuli should have different 
meanings in PTSD-remittance research. For example, Rooij et al. used trauma-unrelated emotional stimuli and found no difference in the hippocampus and ventromedial prefrontal cortex (vmPFC), which are often implicated in PTSD, between remitted and persistent PTSD veterans.(van Rooij, Kennis, Vink, \& Geuze, 2016) This result may be because these two regions were more involved in fear extinction recall. (Rougemont-Bucking et al., 2011) Trauma-unrelated conditions cannot measure traumatic fear-related processes, while trauma-related stimuli would induce negative emotion and trauma-specific fear in PTSD patients.(van Rooij et al., 2016) However, the trauma-specific component should be particularly meaningful in the PTSD-remittance mechanism because most effective therapies are trauma-focused, such as cognitive-behavioral therapy (CBT),(Hinton, Hofmann, Pollack, \& Otto, 2009) eye movement desensitization and reprocessing (EMDR),(Mavranezouli et al., 2020) and prolonged exposure therapy (PE).(Helpman et al., 2016; Maguen et al., 2019) To study this critical component for remittance, traumarelated stimuli must be applied, and more trauma-specific brain activity may be reflected in the contrast of trauma-related vs. unrelated conditions. However, no research used both conditions. A recent metaanalysis found that trauma-exposed controls and PTSD patients exhibited hyperactivation of the amygdala, but no significant difference was found between these two groups, which indicates that this pattern of activation may not be pathological.(Patel, Spreng, Shin, \& Girard, 2012) Therefore, in studies using a trauma-related stimulus, the use of traumatized controls may help focus on the unique characteristics of PTSD rather than neural markers of trauma exposure.

Therefore, the current experiment studied the unique remittance mechanism of pediatric PTSD via measurement of the corresponding neural activities of youths who remitted from PTSD, persistent PTSD youths and traumatized controls 19 months after an earthquake using earthquake pictures (trauma related) and trauma-unrelated negative emotional pictures. We used whole-brain analysis instead of predefined region of interests (ROI) because there are only a few related studies, with high heterogeneity. In addition, we also take into consideration that predefined ROls may lead to overrepresentation in some brain regions (e.g., both a recent review(Negreira \& Abdallah, 2019) and a meta-analysis(Sprooten et al., 2017) suggest that amygdala activation is common only in studies using ROls, but rarely in whole-brain analysis). Therefore, we hypothesized that, using whole-brain analysis, under the trauma-unrelated and trauma-related conditions, the remitted group would show different brain activity from that of the PTSD group in the brain regions that related to remittance in previous studies:

hippocampus/PCC/mPFC/amygdala; compared with the control group, PTSD patients may have abnormal brain activities in the brain regions related to the classic pathological mechanism of PTSD: hippocampus/mPFC/amygdala; there is no significant difference between the remitted group and control group. In trauma-related vs. trauma-unrelated contrast, the PTSD group may exhibit different brain activities, while there would be no significant differences in the remitted group.

\section{Methods}

\subsection{Participants}


All participants were 8- to 18-year-old youths who survived the 2008 Wenchuan earthquake in China. Some of the behavioral data of the participants in the current research were included in our previous study.(R. Yang et al., 2014) In the current fMRI experiment, our psychiatric clinicians interviewed all participants according to the Present and Lifetime version of the Schedule for Affective Disorders and Schizophrenia for School-Age Youth (K-SADS-PL)(Kaufman, Birmaher, Brent, Ryan, \& Rao, 2000) 17 months after the earthquake, but the fMRI scan was applied at 19 months because of the complex situation after the disaster. The following inclusion criteria were used for the PTSD group: (a) earthquakeexposed youths age between 8-17 years at the time point of the trauma; (b) diagnosed as current PTSD by psychiatric clinicians according to the K-SADS-PL(Kaufman et al., 2000) 17 months after the earthquake; and (c) right-handed. The following exclusion criteria were used: (a) other Axis-I psychiatric diagnosis, except comorbid mood/anxiety disorders for the PTSD group; (b) IQ < 80 using the Chinese Wechsler Intelligence Scale for Children (C-WISC)(Gong \& Cai, 1993); (c) use of psychotropic medications in the past 4 weeks; and (d) any significant medical or neurological conditions or a history of head injury. The inclusion and exclusion criteria of the remitted and control groups were similar to those of the PTSD group, except that the remitted participants met the diagnosis of lifetime PTSD, but not current PTSD, in the 17th month interview, and the controls never met the diagnosis criteria of any psychiatric diagnosis. Twenty-five healthy controls, 15 PTSD patients and 23 remitted participants were recruited in our fMRI study. Data sets were also excluded for excessive head motion (translations and rotations were larger than $2.5 \mathrm{~mm}$ and 2.5 degrees) and poor accuracy on the task (over $10 \%$ missing). Participants included in the final analysis consisted of 18 healthy, 13 PTSD and 18 remitted youths. Among the 13 participants with PTSD, two of them received EMDR, one participant received EMDR and CBT, one received sertraline for 1 month, and one received clonidine controlled-release patches 4 times. No medication was applied within four weeks before scanning. According to the K-SADS interview, none of the subjects had any other experience of traumatic stress events except this earthquake.

The Institutional Review Board at the Health Center of Peking University approved this study. Written informed consents were obtained from each participant and their guardians.

\subsection{Affective Processing Task}

During fMRI scan, participants performed a block-designed affective processing task with earthquakerelated/unrelated emotional pictures and scrambled pictures as baseline. One trial consisted of a 4.5second picture and a 0.5 -second black screen. There were 6 trials in each block. The blocks were arranged in a fixed order: $+\mathrm{S}+\mathrm{N}+\mathrm{E}+\mathrm{S}+\mathrm{N}+\mathrm{E}+\mathrm{S}+\mathrm{N}+\mathrm{E}+(+$, rest; $\mathrm{S}$, scrambled picture; $\mathrm{N}$, trauma unrelated negative picture; $\mathrm{E}$, trauma related earthquake picture). A relatively long rest time between blocks (20 s) was adopted to avoid anxiety elicited by the trauma pictures from persisting into the trauma-unrelated pictures. All participants were asked to press the thumb button when they saw a picture appear to ensure that they were actually watching. Participants who did not respond to more than $10 \%$ of pictures were excluded.

Eighteen pictures depicting the Wenchuan earthquake were collected from the internet and primarily portrayed collapsed buildings in Wenchuan with dead or wounded civilians. Eighteen negative but 
unrelated-to-earthquake-scene pictures were selected from the International Affective Picture System (IAPS,(Jayaro, de la Vega, Diaz-Marsa, Montes, \& Carrasco, 2008) which has been widely used in PTSD research(Negreira \& Abdallah, 2019)) and included depictions of diseases, poverty, filth, fire, violent assaults and horrible faces without any collapsed buildings. Two groups of pictures were balanced in emotional valence and arousal, as measured with a Self-Assessment Manikin (SAM)(Bynion \& Feldner, 2017). Examples of the pictures are shown in Fig. 1. Supplement 1, which is available online, provides the details of the evaluation and screening of pictures, the making of scrambled pictures and the properties of images. Each group of pictures was randomly divided into three blocks.

\section{3. fMRI Data Acquisition}

Supplement 1, available online, provides details.

\section{4. fMRI Data Analysis}

Preprocessing was performed using Statistical Parametric Mapping (SPM12, http://www.fil.ion.ucl.ac.uk/spm) and DPABI: a toolbox for Data Processing \& Analysis for Brain Imaging(Yan, Wang, Zuo, \& Zang, 2016). Supplement 1 is available online and provides additional details.

The experimental sequences (trauma pictures vs. scrambled pictures, trauma-unrelated pictures vs. scrambled pictures, trauma pictures vs. trauma-unrelated pictures) were modeled using a hemodynamic response function (HRF)-convolved boxcar model with no derivatives, and global scaling was applied. We first produced between-condition SPMs for each participant. Second, we undertook between-condition contrasts at a within-group level and a between-group level. In the within-group contrasts, voxels of significant activity (trauma-related vs. scrambled, trauma-unrelated vs. scrambled, voxel thresholds $p<$ 0.0001 , cluster thresholds $p<0.005$, one tailed) in all three groups, and voxels with significant different reaction to trauma-related/unrelated stimuli (trauma related vs. trauma unrelated, voxel thresholds $p<$ 0.0001 , cluster thresholds $p<0.005$, two tailed) in the PTSD and remitted groups were determined after thresholding using Gaussian random field (GRF) correction(Chen, Lu, \& Yan, 2018) in the DPABI toolbox. To compare differences in brain activity between the three groups under trauma-related/unrelated conditions, the between-group contrasts were applied in two conditions separately. We generated a union mask of activated brain regions ( $p<0.05$, no correction) in any group and performed ANOVA within the mask with GRF correction (voxel thresholds $p<0.005$, cluster thresholds $p<0.05$ ). Post hoc testing extracted values from resulting clusters that showed a main effect of group in the ANOVA for each participant and analyzed between-group differences with independent samples t tests.

We also explored the group analysis and within-group analysis on the trauma-related vs trauma-unrelated contrast, as well as correlation analysis in brain regions to study the relationship between brain activity.

\section{Results}

\subsection{Demographic Data}


The information for the demographics/clinical data of the participants is summarized in Table 1. The differences in gender, age, and IQ were not significant.

Table 1

Demographics/Clinical Information of Participants

\begin{tabular}{|c|c|c|c|c|c|}
\hline Group & Healthy & PTSD & Remitted & Statistics & \\
\hline & $(n=18)$ & $(n=13)$ & $(n=18)$ & $F / \chi^{2}$ & $p$ \\
\hline \multirow[t]{2}{*}{ Age (years) } & $15.21(1.63)$ & $15.21(2.50)$ & $15.23(1.93)$ & $\begin{array}{l}F(2,46)= \\
0.001\end{array}$ & 0.999 \\
\hline & $\begin{array}{l}\text { range: } 12.1 \sim \\
18.1\end{array}$ & $\begin{array}{l}\text { range: } 8.9 \sim \\
18.1\end{array}$ & \multicolumn{3}{|l|}{ range: $10.4 \sim 18.3$} \\
\hline Gender(M: F) & $10: 8$ & $4: 9$ & $6: 12$ & $\chi^{2}=2.579$ & 0.275 \\
\hline \multirow[t]{2}{*}{ IQ } & $106.67(12.00)$ & $108.85(14.46)$ & $\begin{array}{l}102.39 \\
(10.46)\end{array}$ & $\begin{array}{l}F(2,46)= \\
1.156\end{array}$ & 0.324 \\
\hline & range: $80 \sim 125$ & range: $83 \sim 129$ & \multicolumn{3}{|l|}{ range: $84 \sim 127$} \\
\hline Comorbidities & None & $3 \mathrm{MDD}$ & $1 \mathrm{MDD}$ & & $<0.001$ \\
\hline
\end{tabular}

Activation patterns for each group under the trauma-unrelated condition are shown in Fig. 2A. The significantly activated cluster was primarily located in the bilateral middle occipital gyrus. The control and remitted groups showed activated bilateral hippocampus, and the PTSD group showed no significant activation in that area. ANOVA revealed that the brain region located in the left hippocampus/parahippocampus $(-21-12-21 ; k=19)(F i g .2 b)$ exhibited a significant main effect of group. However, when we tried a relatively strict correction (GRF correction, voxel thresholds $p<0.005$, cluster thresholds $p<0.005$ ), this result was not significant. Post hoc analysis showed that the brain activity of this cluster in the remitted group was higher than in the PTSD group and lower than in the controls. There were significant differences between the three groups (Fig. 2c).

\subsection{Trauma-related Brain Responses in the Three Groups}

Activation patterns for each group under trauma-related conditions are shown in Fig. 3A. The patterns of the three groups were similar and included the bilateral occipital cortex, primarily the middle occipital gyrus and lingual gyrus, and extending to the bilateral temporal lobe and bilateral parahippocampal gyrus. ANOVA revealed that the brain area that exhibited a significant main effect of group was located in the right middle occipital gyrus/precuneus $(36-6924, k=40)$ (Fig. 3b). This result was still significant when a relatively strict threshold was applied (GRF correction, voxel thresholds $p<0.005$, cluster thresholds $p<0.005)$. Post hoc analysis showed that brain activity in the PTSD group and remitted group 
was significantly higher than in the control group, but there was no difference between the PTSD and remitted groups (Fig. 3c). When we tried to use the cluster in the left hippocampus from the previous analysis as a mask for ANOVA, there was no significant difference among the three groups (GRF correction, voxel thresholds $p<0.05$, cluster thresholds $p<0.05$ ).

\subsection{Neural Activity Difference Between Trauma- Related/Unrelated Conditions}

\subsubsection{PTSD Group: Trauma-Related vs. Trauma-Unrelated}

Surprisingly, we found no significant neural activity difference between the two conditions in the PTSD group after GRF correction (voxel thresholds $p<0.0001$, cluster thresholds $p<0.005$, two-tailed). There was still no significant result when we tried a relatively loose threshold $(p<0.001$, cluster thresholds $p<$ 0.05 , two-tailed).

\subsubsection{Remitted Group: Trauma-Related vs. Trauma- Unrelated}

In the remitted group, trauma-specific pictures elicited a significant increase in neural activity in the right parahippocampal gyrus (33-42 -6; $t=7.01 ; k=43$ voxels) and right lingual gyrus $(12-96-6 ; t=7.39 ; k=$ 97 voxels) compared to the trauma-unrelated condition (Fig. 4).

\subsection{Exploratory Analysis}

\subsubsection{Between-Group Comparison in Trauma- Related/Unrelated Contrast}

No significant group effect was found in the whole-brain analysis (voxel thresholds $p<0.005$, cluster thresholds $p<0.05$ ). When we conducted analysis in the mask of four brain regions with positive results in the above analysis separately (right middle occipital gyrus, left hippocampus, right parahippocampal gyrus and right lingual gyrus), we found that only the left hippocampus survived after correction (GRF correction voxel $p<0.0005$, cluster $p<0.0005$, post hoc: control $<$ remnant $=\mathrm{PTSD}$ ). To visually show the relative and absolute differences of brain activity in three groups under different conditions, we show the signals extracted from these clusters, as well as the results of the between-group comparison. (Fig. 5)

\subsubsection{Control Group: Trauma-Related vs. Trauma-Unrelated}

See Supplement.

\subsubsection{Correlation Between Left Hippocampus Brain Activities under the Trauma-Unrelated Condition and Signal}




\section{Change Between Conditions}

See Supplement.

\subsubsection{Group Analysis with Age/Age + Gender + Comorbidity as Covariates}

See Supplement.

\section{Discussion}

The current study focused on the brain mechanism of remitted PTSD children and adolescents after earthquake compared with current PTSD and a trauma-exposed control using trauma-unrelated and trauma-related stimuli. As hypothesized, in the comparison between the PTSD group and the remitted group, under the trauma-unrelated condition, PTSD exhibited lower activity in the left hippocampus. However, no significant difference was found under the trauma-related condition. In the comparison between the PTSD group and the control group, PTSD exhibited lower activity in the left hippocampus under the trauma-unrelated condition and higher activity in the right middle occipital gyrus under the trauma-related condition. There was a similar pattern in the comparison between the remitted group and the control group, but the activity of the left hippocampus of the remitted group was in the middle between the other groups under the trauma-unrelated condition. In trauma-related vs. trauma-unrelated contrast, contrary to our hypothesis, there was no significant neural activity difference in the PTSD group, while in the remitted group, different brain activity was found in the right parahippocampal gyrus and right lingual gyrus. We also explored a group analysis of trauma-related vs. trauma-unrelated contrast and found that the activity difference in the left hippocampus was PTSD = removed $>$ control. No difference in $\mathrm{mPFC} / \mathrm{ACC} /$ amygdala was found in any comparison.

The pathological mechanism of PTSD is closely related to the hippocampus. The largest PTSD brain image study included 1868 participants from 16 cohorts confirmed the relationship between PTSD and a smaller hippocampus volume.(Logue et al., 2018) Klaming et al. also found the correlation between right hippocampus morphology and symptom severity in 70 trauma-exposed veterans.(Klaming, Spadoni, Veltman, \& Simmons, 2019) Furthermore, in 2017, Malejko et al. reviewed the research on PTSD remission and found that the remission of PTSD was often accompanied by a change in hippocampus activity.(Malejko et al., 2017) In the current study, using whole-brain analysis, the only significant difference between the PTSD group and the remitted group was in the left hippocampus under the trauma-unrelated condition. To be specific, the remitted group exhibited higher activity than the PTSD group, and lower than the control group. This finding supported the significance of the hippocampus, as well as trauma-unrelated stimuli, in the remittance mechanism of PTSD. In the following exploratory analysis, we also found that this cluster was the only one with inter-group differences under traumarelated vs trauma-unrelated contrast. 
A number of studies have found that the volume of the occipital lobe in the PTSD population is smaller than that of the control group,(Cwik et al., 2019; Sussman, Pang, Jetly, Dunkley, \& Taylor, 2016) In adult(Gudrun et al., 2013; Ke et al., 2015) and children(P. Yang, Wu, Hsu, \& Ker, 2004) PTSD studies, this region also showed abnormal high activity under trauma-related conditions. This may be due to the involvement of the occipital lobe in the transformation of traumatic memory in visual form into narrative trauma-related memories.(Lanius, Bluhm, Lanius, \& Pain, 2006) In the current study, the hyperactivity in right middle occipital gyrus in the PTSD and remitted group may reflect the re-experiencing under traumarelated stimuli condition.

Contrary to our intuition, the remitted individuals still had PTSD-like brain responses to trauma stimuli. This state-independent feature may be interpreted as the endophenotype of PTSD.(McAuley, Crosbie, Charach, \& Schachar, 2014) However, there is still a question from the other side of this phenomenon: how can we define an individual who still exhibits 'abnormal' brain activity under trauma stimuli as remitted? This dichotomy may occur because our definition of the state of psychiatric disorder highly depends on the influence or potential influence of symptoms on an individual's social function, and the 'abnormal' brain activity that existed only during trauma-specific stimulation had relatively little influence on social function. The PTSD diagnostic criterion G in DSM-5 is 'The disturbance causes clinically significant distress or impairment in social, occupational, or other important areas of functioning', which directly points to the evaluation of social functions.(Bovin et al., 2015) During the K-SADS interview, psychiatric clinicians do not simply judge the presence or absence of a symptom but spend a large proportion of time verifying the possible impact of suspicious symptoms on patients' functions. If an individual only have 'abnormal response' to stimuli that are highly specific to the original trauma (traumarelated stimulus), then the chance of exposure in daily life to cause functional damage will be relatively low, and the patient will consequently be defined as 'remitted', even if the possibility of an abnormal response under very special circumstances exists. Schnurr et al. studied the relationship between symptom improvement benchmarks and the quality of life of PTSD patients and found that remission (defined as loss of diagnosis plus a severity score $<20$ in the Clinician-Administered PTSD Scale) did not yield more benefit than the loss of diagnosis, although remission is considered the most desirable outcome for relieving PTSD symptom burden.(Schnurr \& Lunney, 2016) This result is not direct evidence, but it is also supported by results that the loss of diagnosis in PTSD patients does not always mean complete 'normalization' at the symptom level, but more meaningfully at the level of social function. Similar to the current finding, participants who were defined as remitted did not achieve complete 'normalization'.

There could be a potential variable related to social function of earthquake induced PTSD - the "Commonness" of the trauma, referencing the probability of exposure to related cues in daily life. In the current study, earthquake-related cues should be relatively rare in daily life, and correspondingly, the chance to affect individuals was low. Frequent stimulation does cause pain to individuals, but also provides more opportunities for extinguish learning. The 'Commonness' of cues in PTSD should be a factor between the dynamic balance of persist-remit and a potential difference between various traumas. Therefore, 'commonness' could be one direction for future research. 
In the remitted group, the right parahippocampal gyrus and the right lingual gyrus exhibited higher brain activity under the trauma-related condition than under the trauma-unrelated condition. These clusters were highly consistent with the findings from another earthquake-induced PTSD study on youths, in which PTSD youths exhibited higher activity than controls in the same brain regions only.(P. Yang et al., 2004) The parahippocampal gyrus is involved in the storage and retrieval of emotional memory.(P. Yang et al., 2004) And lingual gyrus is related to narrative memory.(Lanius et al., 2006) In previous study, reduction of the lingual gyrus is related to the severity of PTSD symptoms.(Wrocklage et al., 2017) The current findings in the remitted group may also reflect the re-experiencing under trauma condition, and there is no such response under the trauma-unrelated condition.

In contrast, there is no significant brain activity difference in the PTSD group between the two conditions. This result, however, needs to be discussed very carefully. We previously considered whether this statistically negative result could be a supportive evidence for the claim in previous studies that abnormalities of PTSD patients are "generalized" to trauma unrelated condition.(van Rooij et al., 2015; Zinchenko et al., 2017) However, inspection of Fig. 5 shows that this trauma related vs. trauma unrelated difference between the groups is rather small, and was not statistically significant. Exploratory correlation analysis also failed to find a significant correlation between brain activity and generalization effect. In the future, bigger sample with better statistical power will be necessary to be applied to verify these findings. In addition, if researchers want to study the generalization effect, a more specific material should be considered, such as hieratically larger circles, to get a more convincing conclusion.

The current findings support that trauma-unrelated negative stimuli, rather than trauma stimuli, play an important role in PTSD remission. However, most effective therapies for PTSD are trauma-focused. (Helpman et al., 2016; Hinton et al., 2009; Maguen et al., 2019; Mavranezouli et al., 2020) Therefore, negative emotional stimuli (with high commonness) must be taken into account in future therapies. Kaczkurkin et al. also argued that because of the important role of maladaptive generalization in the pathological mechanism of PTSD, future psychotherapy may also involve trauma-like stimulation (generalized stimulation).(Kaczkurkin et al., 2017) Therefore, nontrauma-focused therapy may a future direction of PTSD treatment.

We did not find differences between groups in PFC/ACC or amygdala, which were reported in previous PTSD-remittance studies.(Cisler et al., 2015; Negreira \& Abdallah, 2019) This discrepancy may be caused by differences in age, gender, trauma experience, use of ROI and the paradigms applied.(Malejko et al., 2017; Negreira \& Abdallah, 2019) A previous PTSD study using trauma-related stimulus found different brain activities in the amygdala and ACC in different genders.(Shin et al., 2004) In the current study, the mixed genders of subjects may erase the effect of some brain regions, especially the amygdala and mPFC. Furthermore, the classic pathological model of PTSD is mostly based on adult studies. However, there should be differences between youths and adults.(Herringa, 2017) Thus, the findings in PTSD research in youths could be different from the classical theory. In addition, considering the special original trauma (earthquake) in the current study, and thus differences in paradigm/stimulus, the results will also be affected. Interestingly, the brain regions revealed by the current study are similar to the only 
PTSD study on mixed gender youths after an earthquake, which included occipital lobe, hippocampus/parahippocampus, but no prefrontal lobe/ACC (earthquake).(P. Yang et al., 2004)

Our study had several limitations. First, a small sample size was used due to the common difficulties of task-state fMRI studies on natural disaster-induced PTSD. Among all 10 previous related studies, the sample sizes of the PTSD groups ranged from 5-16 subjects, with an average of 10.9 subjects.(Du et al., 2014; Piccardi et al., 2016; P. Yang et al., 2004) Small groups provide relatively low statistical power and make it difficult to strictly control the comorbidity and treatment of subjects; therefore, expanded sample size and controlled comorbidity and treatment are needed in the future to validate the findings of the current study. Second, the statistical thresholding was liberal, which increased the risk for false-positive results. However, it also makes our negative findings more robust, such as the similarity of brain activities between the remitted and PTSD groups under trauma-related conditions. In addition, the P value of GRF correction in this study is less than 0.01 , which is enough to make the false-positive rate lower than 0.05 , even if the spatial distribution does not satisfy the Gaussian distribution.(Eklund, Nichols, \& Knutsson, 2016) Third, the generalizability of the current findings may be limited because of the particularity of earthquake-induced PTSD. Previous studies showed that the type of original trauma affected the heterogeneity of PTSD research.(Boccia et al., 2016; Negreira \& Abdallah, 2019) The low "Commonness" of earthquake-specific stimuli may also have a potential impact on the remittance processing of PTSD patients. Future PTSD experiments should measure the "Commonness" of stimuli and study their influence on PTSD. Fourth, this study did not include the questionnaires commonly used in PTSD research, such as the childhood trauma questionnaire (CTQ).(Jiang et al., 2018) Future study should consider multiple questionnaires for a comprehensive evaluation. Last, the earthquake pictures used in this study are from Wenchuan. Although there are no landmark buildings and the ruins are difficult to identify (Fig. 1), there may still be a component of personal relevant experiences. In contrast, the negative pictures from IAPs do not have this component, which could be an issue. However, in some previous studies, similarly, the picture stimulus from the particular original trauma was adopted for symptom provocation, such as the Vietnam war rather than other wars,(Wolf, Miller, \& McKinney, 2009) and the mine accidents that subjects experienced instead of other mine accidents.(Hou et al., 2007) Elements related to personally relevant experiences in such pictures may also be types of trauma cues. For example, a trauma cue could be "war-related" or "Vietnam-related" or the combination of both. For the current study, in the between-group comparison, since all the subjects came from Wenchuan, this factor may have relatively little influence. However, it may still complicate comparisons between trauma-related and trauma-unrelated stimuli, especially for the hippocampus, which is responsible for episodic memory. Therefore, in future research, trauma pictures from other regions should be adopted to exclude the potential impact of personally relevant experiences. Moreover, instead of scrambled pictures, future studies could adopt pictures depicting people and/or buildings that are as neutral as possible as a control condition to distinguish between general object recognition processes and more specific affective processes. Furthermore, presentation of stimulus in a fixed order may lead to a risk of systematic spillover effects. Thus, (quasi-)randomization of blocks or trials could be considered in future studies. 


\section{Declarations}

\section{Ethics approval and consent to participate}

The Institutional Review Board at the Health Center of Peking University approved this study.

\section{Consent for publication}

Written informed consents were obtained from each participant and their guardians.

\section{Availability of data and material}

The datasets during and/or analysed during the current study available from the corresponding author on reasonable request.

\section{Competing interests}

The authors declare that they have no competing interests.

\section{Funding}

This study was funded by the National High Technology Research and Development Program of China (863 Program)(No. 2008AA022605), the National Basic Research Development Program of China (973 program 2014CB846104), the National Natural Sciences Foundation of China (No. 81000594), and the Sanming Project of Medicine in Shenzhen "The ADHD research group from Peking University Sixth hospital"(SZSM201612036).

\section{Authors' contributions}

Conception and design: YW, ZP, YL, QC, LS

Analysis and interpretation: PW, ZP, LL, QC, BY, YW

Data collection: ZP, LL, LA, YL, QC, LS, NJ, YC, YW

Writing the manuscript: PW, ZL

Critical revision of the article: PW, QC

Statistical analysis: PW

Overall responsibility: YW

\section{Acknowledgments}

Thanks are due to Meilin Wang for kind assistance in figure editing and valuable discussion on results. 


\section{References}

1. Boccia, M., D’Amico, S., Bianchini, F., Marano, A., Giannini, A. M., \& Piccardi, L. (2016). Different neural modifications underpin PTSD after different traumatic events: an fMRI meta-analytic study. Brain Imaging \& Behavior, 10(1), 226-237.

2. Bovin, M. J., Marx, B. P., \& Schnurr, P. P. (2015). Evolving DSM Diagnostic Criteria for PTSD: Relevance for Assessment and Treatment. Current Treatment Options in Psychiatry, 2(1), 1-13.

3. Bynion, T. M., \& Feldner, M. T. (2017). Self-Assessment Manikin: Springer International Publishing.

4. Chen, X., Lu, B., \& Yan, C. G. (2018). Reproducibility of R-fMRI metrics on the impact of different strategies for multiple comparison correction and sample sizes. Hum Brain Mapp, 39(1), 300-318. doi:10.1002/hbm.23843

5. Cisler, J. M., Sigel, B. A., Kramer, T. L., Smitherman, S., Vanderzee, K., Pemberton, J., \& Kilts, C. D. (2015). Amygdala response predicts trajectory of symptom reduction during Trauma-Focused Cognitive-Behavioral Therapy among adolescent girls with PTSD. J Psychiatr Res, 71, 33-40. doi:10.1016/j.jpsychires.2015.09.011

6. Cisler, J. M., Sigel, B. A., Kramer, T. L., Smitherman, S., Vanderzee, K., Pemberton, J., \& Kilts, C. D. (2016). Modes of Large-Scale Brain Network Organization during Threat Processing and Posttraumatic Stress Disorder Symptom Reduction during TF-CBT among Adolescent Girls. PLoS One, 17(8), e0159620. doi:10.1371/journal.pone.0159620

7. Cisler, J. M., Sigel, B. A., Steele, J. S., Smitherman, S., Vanderzee, K., Pemberton, J., . . Kilts, C. D. (2016). Changes in functional connectivity of the amygdala during cognitive reappraisal predict symptom reduction during trauma-focused cognitive-behavioral therapy among adolescent girls with post-traumatic stress disorder. Psychol Med, 46(14), 3013-3023. doi:10.1017/S0033291716001847

8. Cwik, J. C., Vahle, N., Woud, M. L., Potthoff, D., Kessler, H., Sartory, G., \& Seitz, R. J. (2019). Reduced gray matter volume in the left prefrontal, occipital, and temporal regions as predictors for posttraumatic stress disorder: a voxel-based morphometric study. Eur Arch Psychiatry Clin Neurosci. doi:10.1007/s00406-019-01011-2

9. Du, X., Wei, D., Ganzel, B., Kim, P., Zhang, Q., \& Qiu, J. (2014). Adolescent earthquake survivors' show increased prefrontal cortex activation to masked earthquake images as adults. Int J Psychophysiol. doi:10.1016/j.ijpsycho.2014.12.001

10. Eklund, A., Nichols, T. E., \& Knutsson, H. (2016). Cluster failure: Why fMRI inferences for spatial extent have inflated false-positive rates. Proceedings of the National Academy of Sciences, 113(28), $7900-$ 7905. doi:10.1073/pnas.1602413113

11. Garrett, A., Cohen, J. A., Zack, S., Carrion, V., Jo, B., Blader, J., .. Agras, W. S. (2019). Longitudinal changes in brain function associated with symptom improvement in youth with PTSD. J Psychiatr Res, 114, 161-169. doi:10.1016/j.jpsychires.2019.04.021

12. Gong, Y., \& Cai, T. (1993). Wechsler Intelligence Scale for Chinese Children (C-WISC). Changsha: Hunan Map Press. 
13. Gudrun, S., Jan, C., Helge, K., Benjamin, S., Morena, L., Seitz, R. J., \& Ralf, S. (2013). In search of the trauma memory: a meta-analysis of functional neuroimaging studies of symptom provocation in posttraumatic stress disorder (PTSD). PLoS One, 8(3), e58150.

14. Helpman, L., Marin, M. F., Papini, S., Zhu, X., Sullivan, G. M., Schneier, F., . . Neria, Y. (2016). Neural changes in extinction recall following prolonged exposure treatment for PTSD: A longitudinal fMRI study. Neuroimage Clin, 12, 715-723. doi:10.1016/j.nicl.2016.10.007

15. Herringa, R. J. (2017). Trauma, PTSD, and the Developing Brain. Curr Psychiatry Rep, 19(10), 69. doi:10.1007/s11920-017-0825-3

16. Hinton, D. E., Hofmann, S. G., Pollack, M. H., \& Otto, M. W. (2009). Mechanisms of efficacy of CBT for Cambodian refugees with PTSD: improvement in emotion regulation and orthostatic blood pressure response. CNS Neurosci Ther, 15(3), 255-263. doi:10.1111/j.1755-5949.2009.00100.x

17. Hou, C., Liu, J., Wang, K., Li, L., Liang, M., He, Z., .. . Jiang, T. (2007). Brain responses to symptom provocation and trauma-related short-term memory recall in coal mining accident survivors with acute severe PTSD. Brain Res, 1144, 165-174. doi:10.1016/j.brainres.2007.01.089

18. Jayaro, C., de la Vega, I., Diaz-Marsa, M., Montes, A., \& Carrasco, J. L. (2008). [The use of the International Affective Picture System for the study of affective dysregulation in mental disorders]. Actas Esp Psiquiatr, 36(3), 177-182.

19. Jiang, W. J., Zhong, B. L., Liu, L. Z., Zhou, Y. J., Hu, X. H., \& Li, Y. (2018). Reliability and validity of the Chinese version of the Childhood Trauma Questionnaire-Short Form for inpatients with schizophrenia. PLoS One, 13(12), e0208779. doi:10.1371/journal.pone.0208779

20. Kaczkurkin, A. N., Burton, P. C., Chazin, S. M., Manbeck, A. B., Espensen-Sturges, T., Cooper, S. E., . . Lissek, S. (2017). Neural Substrates of Overgeneralized Conditioned Fear in PTSD. Am J Psychiatry, 174(2), 125-134. doi:10.1176/appi.ajp.2016.15121549

21. Kaufman, J., Birmaher, B., Brent, D. A., Ryan, N. D., \& Rao, U. (2000). K-SADS-PL. Journal of the American Academy of Child \& Adolescent Psychiatry, 39(10), 1208-1208.

22. Ke, J., Zhang, L., Qi, R., Xu, Q., Li, W., Hou, C., . . Li, L. (2015). Altered blood oxygen level-dependent signal variability in chronic post-traumatic stress disorder during symptom provocation. Neuropsychiatric Disease \& Treatment, 17(default), 1805-1815.

23. Klaming, R., Spadoni, A. D., Veltman, D. J., \& Simmons, A. N. (2019). Expansion of hippocampal and amygdala shape in posttraumatic stress and early life stress. Neuroimage Clin, 24, 101982. doi:10.1016/j.nicl.2019.101982

24. Lanius, R. A., Bluhm, R., Lanius, U., \& Pain, C. (2006). A review of neuroimaging studies in PTSD: heterogeneity of response to symptom provocation. J Psychiatr Res, 4O(8), 709-729. doi:10.1016/j.jpsychires.2005.07.007

25. Logue, M. W., van Rooij, S. J. H., Dennis, E. L., Davis, S. L., Hayes, J. P., Stevens, J. S., . . Morey, R. A. (2018). Smaller Hippocampal Volume in Posttraumatic Stress Disorder: A Multisite ENIGMA-PGC Study: Subcortical Volumetry Results From Posttraumatic Stress Disorder Consortia. Biol Psychiatry, 83(3), 244-253. doi:10.1016/j.biopsych.2017.09.006 
26. Maguen, S., Holder, N., Madden, E., Li, Y., Seal, K. H., Neylan, T. C., . . Shiner, B. (2019). Evidencebased psychotherapy trends among posttraumatic stress disorder patients in a national healthcare system, 2001-2014. Depress Anxiety. doi:10.1002/da.22983

27. Malejko, K., Abler, B., Plener, P. L., \& Straub, J. (2017). Neural Correlates of Psychotherapeutic Treatment of Post-traumatic Stress Disorder: A Systematic Literature Review. Front Psychiatry, 8(8), $85-$.

28. Mavranezouli, I., Megnin-Viggars, O., Daly, C., Dias, S., Stockton, S., Meiser-Stedman, R., .. Pilling, S. (2020). Research Review: Psychological and psychosocial treatments for children and young people with post-traumatic stress disorder: a network meta-analysis. J Child Psychol Psychiatry, 61(1), 1829. doi:10.1111/jcpp.13094

29. McAuley, T., Crosbie, J., Charach, A., \& Schachar, R. (2014). The persistence of cognitive deficits in remitted and unremitted ADHD: a case for the state-independence of response inhibition. $J$ Child Psychol Psychiatry, 55(3), 292-300. doi:10.1111/jcpp.12160

30. McLaughlin, K. A., Koenen, K. C., Hill, E. D., Petukhova, M., Sampson, N. A., Zaslavsky, A. M., \& Kessler, R. C. (2013). Trauma exposure and posttraumatic stress disorder in a national sample of adolescents. J Am Acad Child Adolesc Psychiatry, 52(8), 815-830 e814. doi:10.1016/j.jaac.2013.05.011

31. Negreira, A. M., \& Abdallah, C. G. (2019). A Review of fMRI Affective Processing Paradigms Used in the Neurobiological Study of Posttraumatic Stress Disorder. Chronic Stress (Thousand Oaks), 3. doi:10.1177/2470547019829035

32. Patel, R., Spreng, R. N., Shin, L. M., \& Girard, T. A. (2012). Neurocircuitry models of posttraumatic stress disorder and beyond: a meta-analysis of functional neuroimaging studies. Neurosci Biobehav Rev, 36(9), 2130-2142. doi:10.1016/j.neubiorev.2012.06.003

33. Piccardi, L., Boccia, M., Colangeli, S., Bianchini, F., Marano, A., Giannini, A. m., . . D'Amico, S. (2016). Neuro-functional alterations due to PTSD after environmental disasters: fMRI evidence and clinical suggestions. Journal of Psychopathology, 22, 165-171.

34. Rosellini, A. J., Liu, H., Petukhova, M. V., Sampson, N. A., Aguilar-Gaxiola, S., Alonso, J., ... Kessler, R. C. (2018). Recovery from DSM-IV post-traumatic stress disorder in the WHO World Mental Health surveys. Psychol Med, 48(3), 437-450. doi:10.1017/S0033291717001817

35. Rougemont-Bucking, A., Linnman, C., Zeffiro, T. A., Zeidan, M. A., Lebron-Milad, K., RodriguezRomaguera, J., . . Milad, M. R. (2011). Altered processing of contextual information during fear extinction in PTSD: an fMRI study. CNS Neurosci Ther, 17(4), 227-236. doi:10.1111/j.17555949.2010.00152.x

36. Schnurr, P. P., \& Lunney, C. A. (2016). Symptom Benchmarks of Improved Quality of Life in Ptsd. Depress Anxiety, 33(3), 247-255. doi:10.1002/da.22477

37. Shin, L. M., Orr, S. P., Carson, M. A., Rauch, S. L., Macklin, M. L., Lasko, N. B., . . Pitman, R. K. (2004). Regional cerebral blood flow in the amygdala and medial prefrontal cortex during traumatic imagery 
in male and female Vietnam veterans with PTSD. Arch Gen Psychiatry, 61(2), 168-176. doi:10.1001/archpsyc.61.2.168

38. Sprooten, E., Rasgon, A., Goodman, M., Carlin, A., Leibu, E., Lee, W. H., \& Frangou, S. (2017). Addressing reverse inference in psychiatric neuroimaging: Meta-analyses of task-related brain activation in common mental disorders. Hum Brain Mapp, 38(4), 1846-1864. doi:10.1002/hbm.23486

39. Sussman, D., Pang, E. W., Jetly, R., Dunkley, B. T., \& Taylor, M. J. (2016). Neuroanatomical features in soldiers with post-traumatic stress disorder. BMC Neurosci, 17, 13. doi:10.1186/s12868-016-0247-x

40. Tang, B., Deng, Q., Glik, D., Dong, J., \& Zhang, L. (2017). A Meta-Analysis of Risk Factors for PostTraumatic Stress Disorder (PTSD) in Adults and Children after Earthquakes. Int J Environ Res Public Health, 14(12). doi:10.3390/ijerph14121537

41. Tang, W., Zhao, J., Lu, Y., Yan, T., Wang, L., Zhang, J., \& Xu, J. (2017). Mental health problems among children and adolescents experiencing two major earthquakes in remote mountainous regions: $\mathrm{A}$ longitudinal study. Compr Psychiatry, 72, 66-73. doi:10.1016/j.comppsych.2016.09.004

42. van Rooij, S. J., Kennis, M., Vink, M., \& Geuze, E. (2016). Predicting Treatment Outcome in PTSD: A Longitudinal Functional MRI Study on Trauma-Unrelated Emotional Processing.

Neuropsychopharmacology, 41(4), 1156-1165. doi:10.1038/npp.2015.257

43. van Rooij, S. J., Rademaker, A. R., Kennis, M., Vink, M., Kahn, R. S., \& Geuze, E. (2015). Neural correlates of trauma-unrelated emotional processing in war veterans with PTSD. Psychol Med, 45(3), 575-587.

44. Wolf, E. J., Miller, M. W., \& McKinney, A. E. (2009). Emotional processing in PTSD: heightened negative emotionality to unpleasant photographic stimuli. J Nerv Ment Dis, 197(6), 419-426. doi:10.1097/NMD.0b013e3181a61c68

45. Wrocklage, K. M., Averill, L. A., Cobb Scott, J., Averill, C. L., Schweinsburg, B., Trejo, M., . . Abdallah, C. G. (2017). Cortical thickness reduction in combat exposed U.S. veterans with and without PTSD. Eur Neuropsychopharmacol, 27(5), 515-525. doi:10.1016/j.euroneuro.2017.02.010

46. Yan, C. G., Wang, X. D., Zuo, X. N., \& Zang, Y. F. (2016). DPABI: Data Processing \& Analysis for (Resting-State) Brain Imaging. Neuroinformatics, 14(3), 339-351.

47. Yang, P., Wu, M. T., Hsu, C. C., \& Ker, J. H. (2004). Evidence of early neurobiological alternations in adolescents with posttraumatic stress disorder: a functional MRI study. Neurosci Lett, 370(1), 13-18.

48. Yang, R., Xiang, Y. T., Shuai, L., Qian, Y., Lai, K. Y., Ungvari, G. S., .. Wang, Y. F. (2014). Executive function in children and adolescents with posttraumatic stress disorder 4 and 12 months after the Sichuan earthquake in China. J Child Psychol Psychiatry, 55(1), 31-38. doi:10.1111/jcpp.12089

49. Zhang, W., Duan, G., Xu, Q., Jia, Z., Bai, Z., Liu, W., .. . Tian, W. (2015). A Cross-sectional Study on Posttraumatic Stress Disorder and General Psychiatric Morbidity Among Adult Survivors 3 Years After the Wenchuan Earthquake, China. Asia Pac J Public Health, 27(8), 860-870.

50. Zinchenko, A., Al-Amin, M. M., Alam, M. M., Mahmud, W., Kabir, N., Reza, H. M., \& Burne, T. H. J. (2017). Content specificity of attentional bias to threat in post-traumatic stress disorder. J Anxiety 
Disord, 50, 33-39.

\section{Figures}

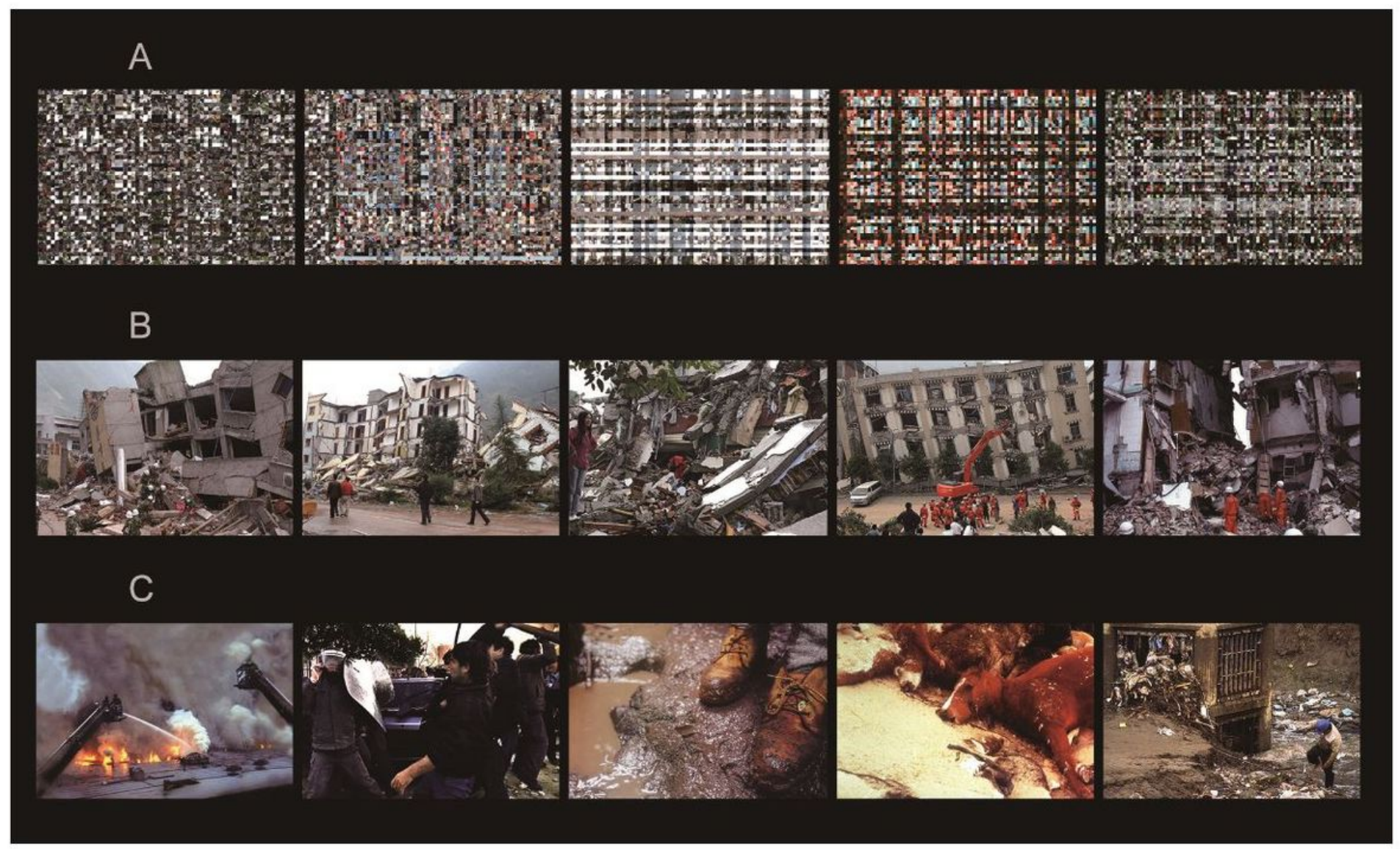

\section{Figure 1}

Example of stimulus pictures. (A) Scrambled pictures. (B) Trauma-related pictures. (C) Trauma-unrelated pictures. 


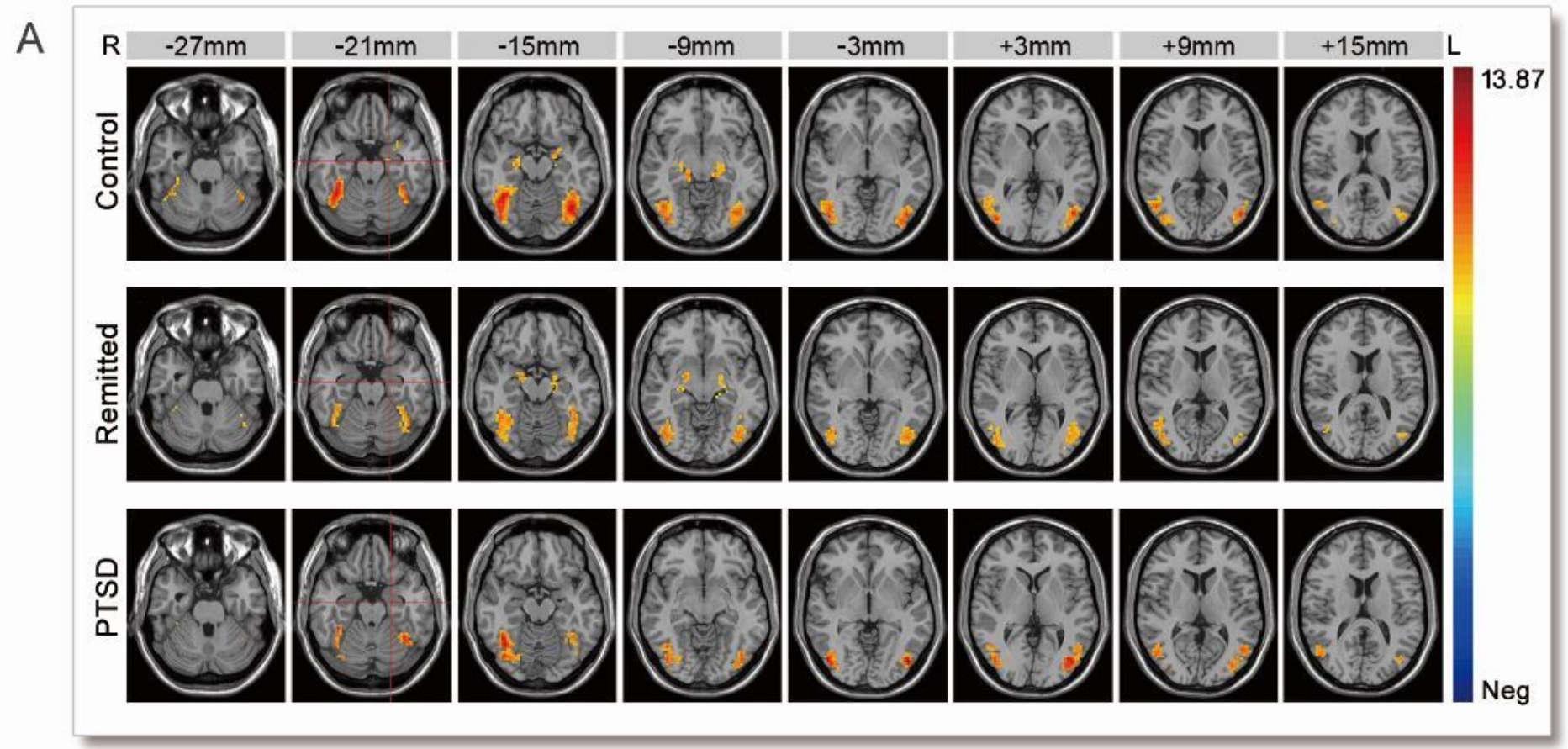

B

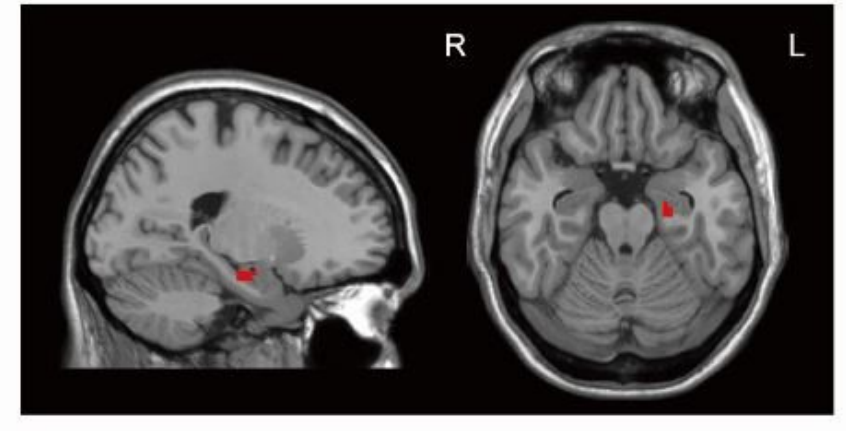

Hippocampus_L (aal): $-21-12-21$

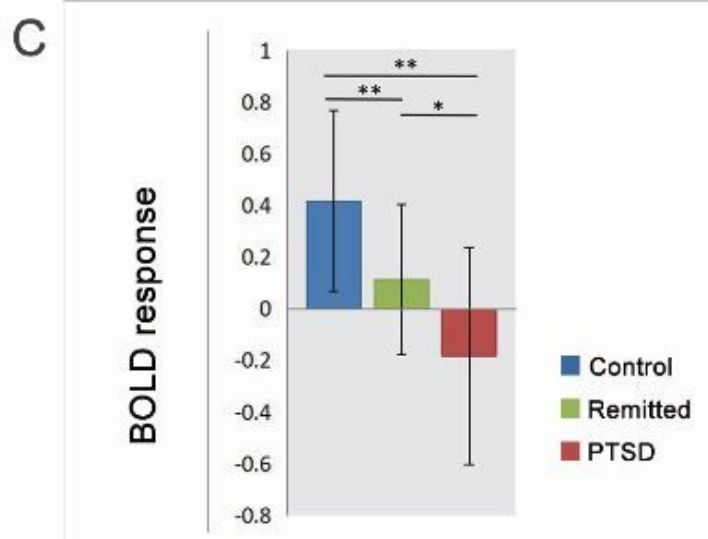

Activation in left hippocampus

Figure 2

Neural activity differences between groups under the trauma-unrelated condition. (A) Trauma unrelated > scrambled activation in the three groups, threshold set at whole-brain GRF correction voxel thresholds $p<$ 0.0001 , cluster thresholds $p<0.005$, one-tailed. (B) Left hippocampus cluster, ANOVA results for the (trauma-unrelated pictures vs. scrambled pictures) contrast within a binary mask by union the three within-group SPMs, with threshold using GRF correction voxel thresholds $p<0.005$, cluster thresholds $p<$ $0.05, k=19$, Peak xyz $=-21-12-21$ (shown in A with red cross). (C) The activities of the three groups in the left hippocampus gyrus cluster. PTSD: posttraumatic stress disorder, BOLD = blood oxygenation leveldependent, ${ }^{\star} p<.05,{ }^{\star *} p<.01$. 

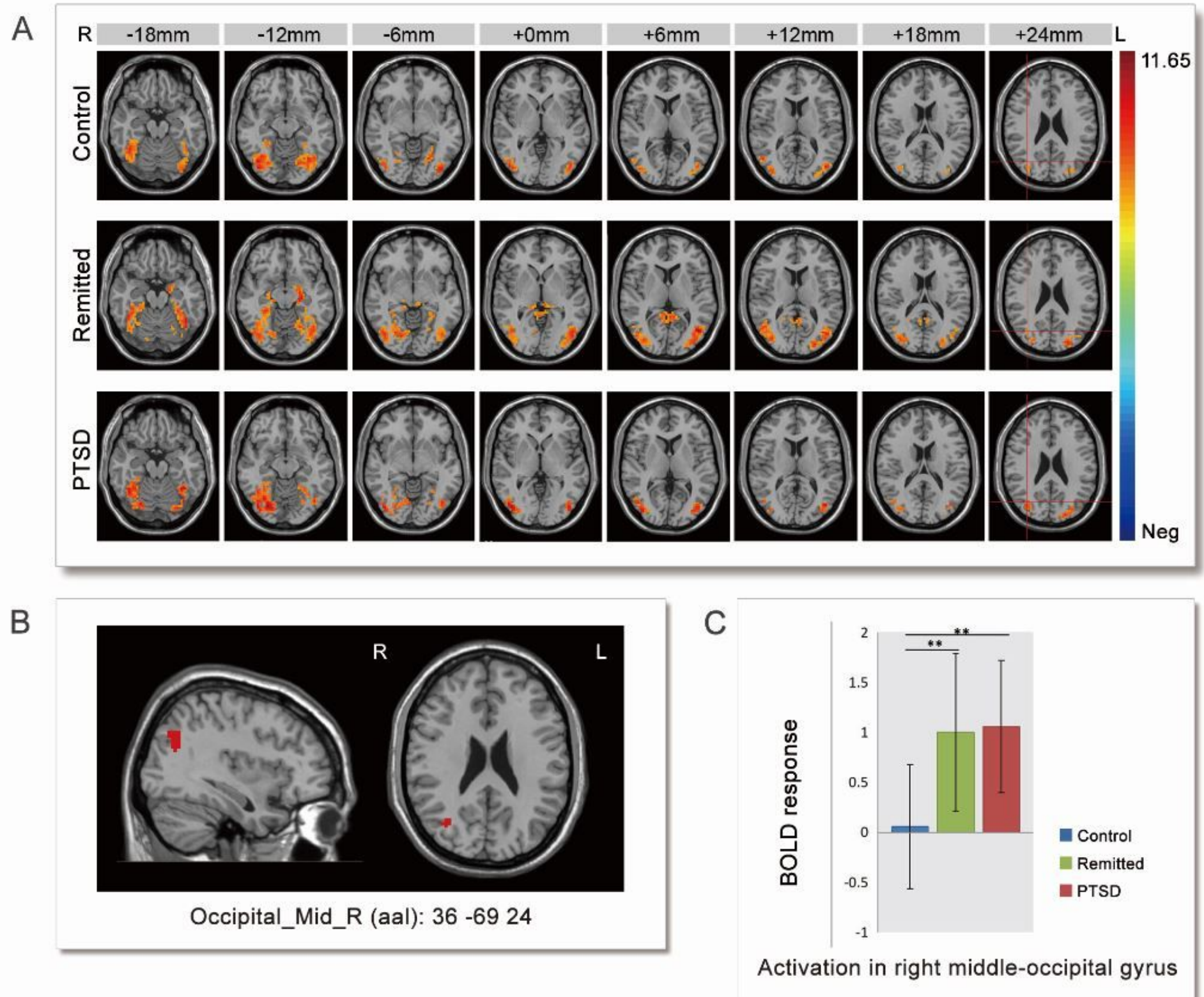

\section{Figure 3}

Neural activity differences between groups under trauma-related conditions. (A) Trauma-related > scrambled activation in the three groups, threshold set at whole-brain GRF correction voxel thresholds $p<$ 0.0001 , cluster thresholds $p<0.005$, one-tailed. (B) Right middle occipital gyrus cluster, ANOVA results for the (trauma-related pictures vs. scrambled pictures) contrast within a binary mask by union of the three within-group SPMs, with a threshold using GRF correction voxel thresholds $p<0.005$, cluster thresholds $p$ $<0.05, k=40$, Peak xyz = $36-6924$ (shown in A with red cross). (C) The activities of the three groups in the right middle occipital gyrus cluster. PTSD: posttraumatic stress disorder, BOLD = blood oxygenation level-dependent, ${ }^{* \star} \mathrm{p}<.01$. 


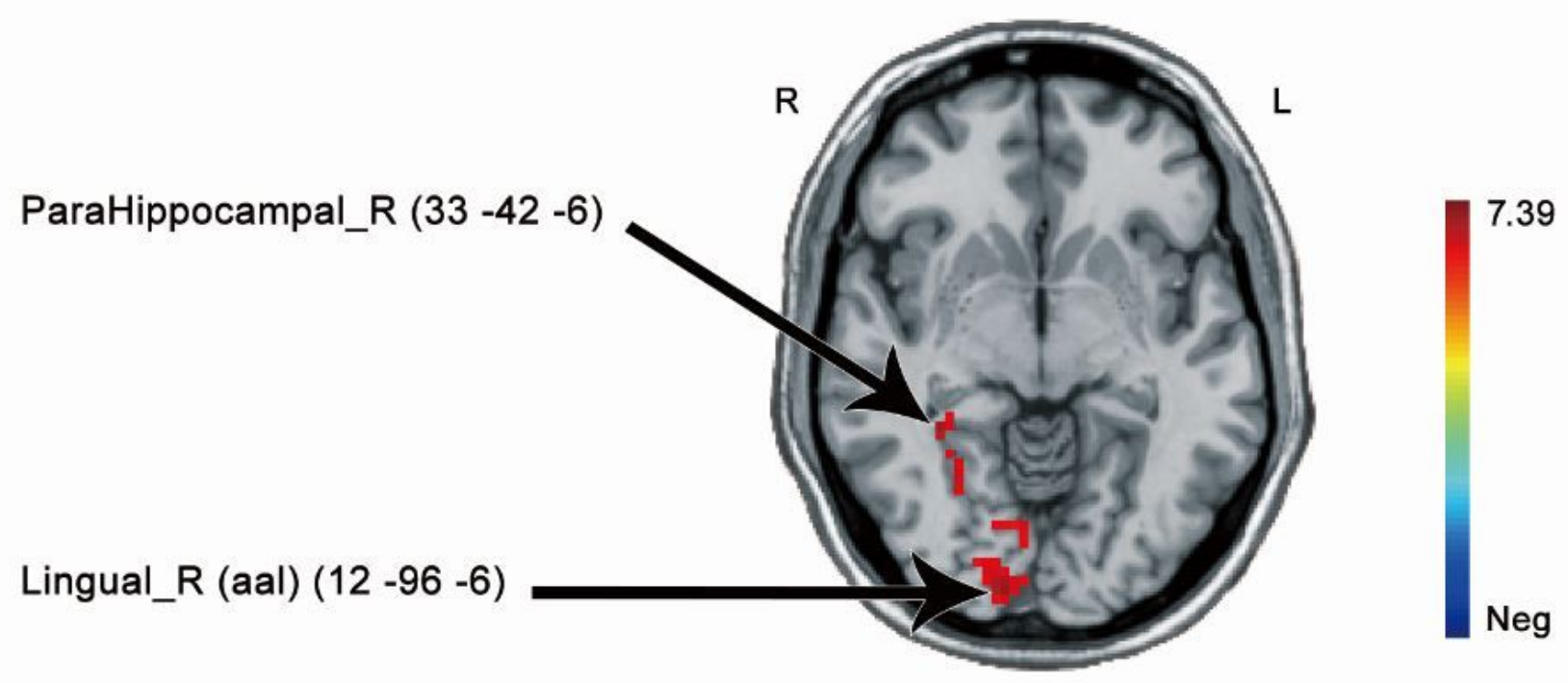

Figure 4

Neural activity differences in trauma-related $>$ trauma-unrelated contrast in the remitted group. Upper cluster $k=43$, Peak xyz = $33-42-6$; Lower cluster $k=97$, Peak xyz = $12-96-6$; threshold set at whole-brain GRF correction voxel thresholds $p<0.0001$, cluster thresholds $p<0.005$, two-tailed. 

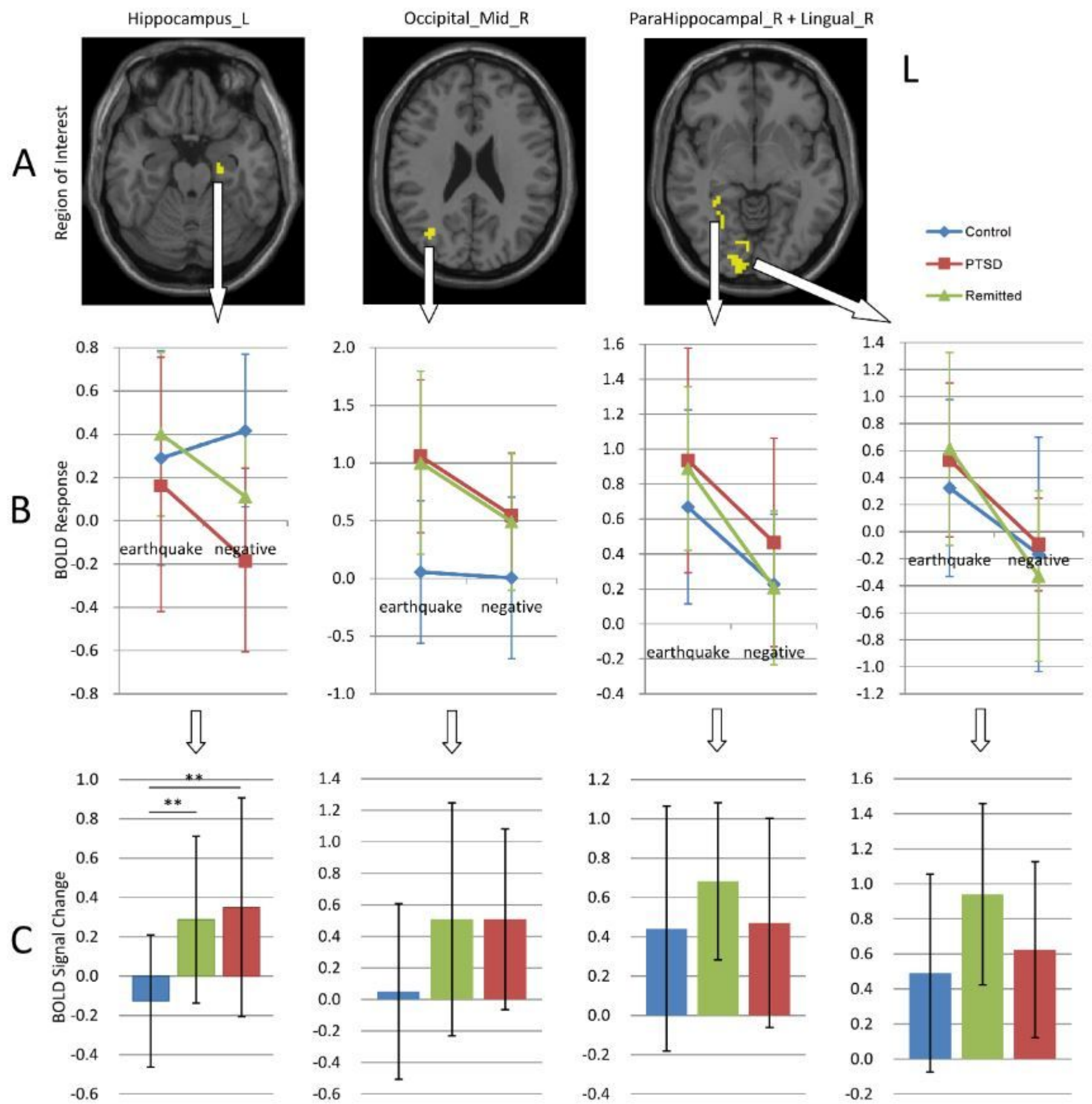

\section{Figure 5}

Neural activity in four clusters under trauma-related and trauma-unrelated conditions. (A) The location of four clusters. (B) The neural activity in three groups under trauma-related (earthquake) and traumaunrelated (negative) conditions. (C) Between-group comparisons of signal change between conditions. PTSD: posttraumatic stress disorder, BOLD = blood oxygenation level dependent, ${ }^{\star \star} p<.01$.

\section{Supplementary Files}


This is a list of supplementary files associated with this preprint. Click to download.

- SupplementFigure4.tif

- SupplementFigure3.tif

- SupplementFigure2.tif

- SupplementFigure1.tif

- WangSupplementaryMaterialforReview.docx 